\title{
Optically nonlinear energy transfer in light-harvesting dendrimers
}

\author{
David L. Andrews ${ }^{\text {a) }}$ and David S. Bradshaw \\ Nanostructures and Photomolecular Systems, School of Chemical Sciences, University of East Anglia, \\ Norwich NR4 7TJ, United Kingdom
}

(Received 27 February 2004; accepted 14 May 2004)

\begin{abstract}
Dendrimeric polymers are the subject of intense research activity geared towards their implementation in nanodevice applications such as energy harvesting systems, organic light-emitting diodes, photosensitizers, low-threshold lasers, and quantum logic elements, etc. A recent development in this area has been the construction of dendrimers specifically designed to exhibit novel forms of optical nonlinearity, exploiting the unique properties of these materials at high levels of photon flux. Starting from a thorough treatment of the underlying theory based on the principles of molecular quantum electrodynamics, it is possible to identify and characterize several optically nonlinear mechanisms for directed energy transfer and energy pooling in multichromophore dendrimers. Such mechanisms fall into two classes: first, those where two-photon absorption by individual donors is followed by transfer of the net energy to an acceptor; second, those where the excitation of two electronically distinct but neighboring donor groups is followed by a collective migration of their energy to a suitable acceptor. Each transfer process is subject to minor dissipative losses. In this paper we describe in detail the balance of factors and the constraints that determines the favored mechanism, which include the excitation statistics, structure of the energy levels, laser coherence factors, chromophore selection rules and architecture, possibilities for the formation of delocalized excitons, spectral overlap, and the overall distribution of donors and acceptors. Furthermore, it transpires that quantum interference between different mechanisms can play an important role. Thus, as the relative importance of each mechanism determines the relevant nanophotonic characteristics, the results reported here afford the means for optimizing highly efficient light-harvesting dendrimer devices. (C) 2004 American Institute of
\end{abstract} Physics. [DOI: 10.1063/1.1769354]

\section{INTRODUCTION}

In a wide range of materials, resonance energy transfer (RET) is the principle mechanism for intermolecular and intramolecular (interchromophore) redistribution of electronic energy following the absorption of ultraviolet/visible radiation. ${ }^{1-3}$ The detailed elucidation of the principles for energy flow in complex systems has led to the devising of new energy-harvesting materials specifically tailored for a host of nanophotonic applications. Chief amongst these new materials are dendrimeric polymers-multiply branched structures of essentially fractal geometry-and other related multichromophore assemblies. ${ }^{4-7}$ Such materials are highly efficient in the capture of optical radiation, as a result of their multiplicity of antenna chromophores and efficient mechanisms for channeling energy to an acceptor core. The applications already range from photodynamic cancer therapy to organic light-emitting diodes.

Recently, attention has begun to focus on dendrimers which exhibit optical nonlinearity. ${ }^{8-12}$ In this connection it has emerged that, in the relevant high intensity regime, suitably designed materials can exhibit two quite different types of mechanism for channeling the excitation energy to an acceptor which is optically transparent at the input (and second harmonic) frequency. Both mechanisms are associated with

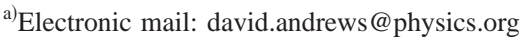

two-photon optical excitation of either a single donor, or a pair of donor chromophores, located close to the acceptor. In the former case the mechanism ${ }^{13,14}$ is a two-photon resonance energy transfer (TPRET) process, initiated by twophoton absorption at a donor, and followed by RET directly to the acceptor. Here the energy transfer can be expressed by the following equation, also schematically illustrated by the energy scheme of Fig. 1:

$$
A^{* *}+B \stackrel{\text { TPRET }}{\longrightarrow} A+B^{*},
$$

where the two-star superscript denotes a two-photon excited state of the donor. The probability for fulfilling the initial conditions for this mechanism (i.e., for the donors to exhibit two-photon absorption) is enhanced at high levels of optical input. In the second case the mechanism is a twin-donor process $^{15}$ which, following initial one-photon excitations of two electronically distinct donors $\left(A\right.$ and $\left.A^{\prime}\right)$, results in an energy pooling, i.e., a collective migration of donor energies to an acceptor chromophore, $B$. Here the more complex RET process is expressible as

$$
A^{*}+B+A^{\prime *} \stackrel{\text { Pooling }}{\longrightarrow} A+B^{*}+A^{\prime}
$$

This mechanism also becomes effective under high intensity laser light due to the enhanced probability of simultaneously exciting two donor chromophores within close proximity of 


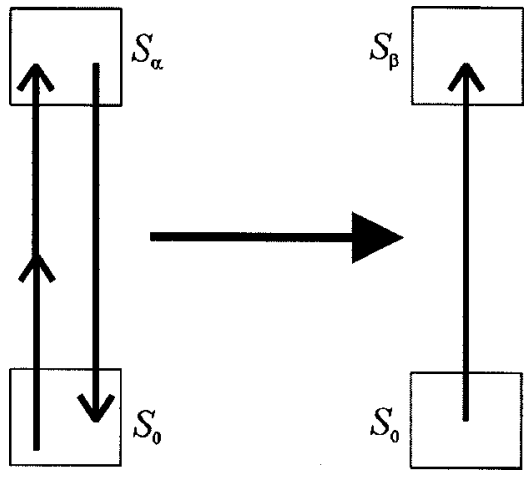

FIG. 1. Energy scheme for TPRET: $S_{0}$ represents the donor ground electronic state and its vibrational manifold; $S_{\alpha}$ denotes a higher electronic state. Vertical arrows represent transitions; horizontal arrows denote channels of excitation.

each other and the acceptor. For both mechanisms it can be assumed experiments are conducted in an ultrafast pumpprobe fashion, so that the system is first subjected to pulsed radiation, and features that exhibit the nonlinear energy transfer are detected subsequently. Further mechanisms that could otherwise lead to the same final state, through the interplay of concurrent energy transfer and pump radiation, are precluded by these experimental conditions (see Sec. V).

To expedite future progress in the development of optically nonlinear light-harvesting systems, it is clearly necessary to ascertain the means of differentiating, optimizing, and exploiting the mechanisms available for energy capture. ${ }^{16-21}$ Since the mechanisms that are available to mediate energy harvesting under conditions of high photon flux differ markedly from those available at lower intensities, it is our aim to secure a thorough understanding of the principles that apply to systems specifically designed for operation at high levels of laser intensity. In this paper we describe in detail the balance of factors and the constraints that determines the favored mechanism for these forms of optical nonlinearity, which include: the excitation statistics, structure of the energy levels, laser coherence factors, chromophore selection rules and architecture, possibilities for the formation of delocalized excitons, spectral overlap, and the overall distribution of donors and acceptors. We begin by eliciting key components of the energy kinetics involved in each mechanism for nonlinear light harvesting.

\section{TWO-PHOTON RESONANCE ENERGY TRANSFER PROCESSES}

A theoretical representation based on molecular quantum electrodynamics (QED) can be used to derive a representation of a complete TPRET process, initiated by two-photon excitation of a single donor. To establish this, we need to consider the detailed nature of the RET step and accommodate statistical features associated with the initial excitation and donor conditions. Determining factors are the number of donors contained within the laser focal volume, the probability of a donor being two-photon excited, and the rate of energy transfer to the acceptor, i.e., the RET step that completes the mechanism. For the ensuing analysis the relevant population factor is simply the number of donors within the focal volume, $N_{A}$ (assuming normal conditions, i.e., below the saturation limit, where ground-state population is very similar to $N_{A}$ ). By defining the number of suitably excited donors within the focal volume as $N_{2 A}$, the probability of two-photon excitation of a donor species under steady-state conditions is

$$
\frac{N_{2 A}}{N_{A}} \approx \frac{k_{A}^{2 \leftarrow 0}}{k_{A}^{0 \leftarrow 2}},
$$

where $k_{A}^{2 \leftarrow 0}$ is the two-photon absorption rate constant of the donor chromophore $A$ (dependent on the input intensity at the appropriate frequency) and $k_{A}^{0 \leftarrow 2}$ is the two-photon deexcitation rate constant. The latter is composed of spontaneous (spon) radiative emission, intramolecular (intra) relaxation, and nonradiative RET rate constants, to give

$$
k_{A}^{0 \leftarrow 2}=k_{\text {spon }}^{0 \leftarrow 2}+k_{\text {intra }}^{0 \leftarrow 2}+k_{\mathrm{RET}}^{0 \leftarrow 2} .
$$

In greater detail, $k_{\text {spon }}^{0 \leftarrow 2}$ includes long-range energy transfer to the "bath" mediated by "real" photons, $k_{\text {intra }}^{0 \leftarrow 2}$ includes internal conversion and intersystem crossing, i.e., repopulation to the ground state by nonradiative or lower energy emission, and $k_{\mathrm{RET}}^{0 \leftarrow 2}$ can be further decomposed to arrive at the expression,

$$
k_{\mathrm{RET}}^{0 \leftarrow 2}=\sum_{n} k_{\mathrm{RET}}^{0 \leftarrow 2}\left(A \rightarrow A_{n}^{\prime}\right)+k_{\mathrm{RET}}^{0 \leftarrow 2}(A \rightarrow B),
$$

where $k_{\mathrm{RET}}^{0 \leftarrow 2}\left(A \rightarrow A^{\prime}\right)$ defines random walk RET to one of $n$, chemically identical neighboring chromophores (the prime denoting exclusion of self-interaction) and $k_{\mathrm{RET}}^{0 \leftarrow 2}(A \rightarrow B)$ is the rate constant for RET from the donor to acceptor species in the near zone. Note that any other "bath" molecules in the near zone are assumed to have sufficiently different optical properties from $A$ and $B$ that they can be excluded from consideration; also stimulated emission is assumed to be negligible. From Eq. (1), $k_{A}^{2 \leftarrow 0}$ is derived by the application of molecular QED leading to the following rotationally averaged result, cast in terms of chromophore properties, ${ }^{22}$

$$
\begin{aligned}
\left\langle k_{A}^{2 \leftarrow 0}\right\rangle= & \frac{\pi\left\langle I^{2}\right\rangle}{120 \hbar}\left(\frac{1}{\epsilon_{0} c}\right)^{2}\left\{\left(2|\mathbf{e} \cdot \mathbf{e}|^{2}-1\right) \alpha_{i i}^{\alpha 0(A)}\left(\omega_{0}, \omega_{0}\right)\right. \\
& \times \bar{\alpha}_{j j}^{\alpha 0(A)}\left(\omega_{0}, \omega_{0}\right)-\left(|\mathbf{e} \cdot \mathbf{e}|^{2}-3\right) \alpha_{i j}^{\alpha 0(A)}\left(\omega_{0}, \omega_{0}\right) \\
& \left.\times \bar{\alpha}_{i j}^{\alpha 0(A)}\left(\omega_{0}, \omega_{0}\right)\right\} \rho_{f}^{A^{* *}} .
\end{aligned}
$$

Equation (4), cast in Cartesian components using the implied summation convention for repeated indices, includes $I$ as the irradiance, $\omega_{0}$ as the optical input circular frequency, $\rho_{f}^{A^{* * *}}$ as the density of states for the excited state, reflecting vibrational broadening, and e.e as the self-product of the laser polarization unit vector, which equates to 0 and 1 for circular and plane polarizations, respectively. Also, $\alpha_{i j}$ is the generalized two-photon response tensor of the general form, ${ }^{22}$

$\alpha_{j k}^{j i(\xi)}\left(\mp \omega_{1}, \mp \omega_{2}\right)=\sum_{\zeta}\left\{\frac{\mu_{j}^{f \zeta(\xi)} \mu_{k}^{\zeta i(\xi)}}{\widetilde{E}_{i \zeta} \pm \hbar \omega_{1}}+\frac{\mu_{k}^{f \zeta(\xi)} \mu_{j}^{\zeta i(\xi)}}{\widetilde{E}_{i \zeta} \pm \hbar \omega_{2}}\right\}$.

In Eq. (5), $i, \zeta$ and $f$ are the initial, virtual and final states, respectively, through which chromophore $\xi$ progresses and 


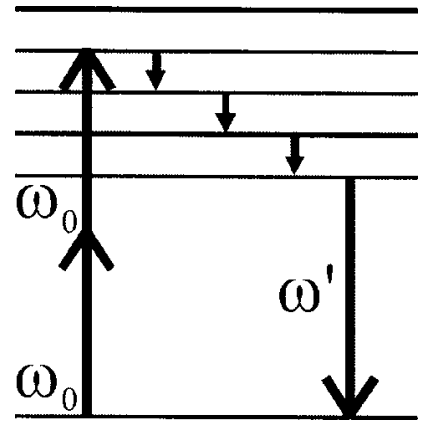

FIG. 2. Nonequivalence of the photon emission, $\omega^{\prime}$, and two-photon absorption, $2 \omega_{0}$, frequencies due to IVR. Large arrows denote electronic transitions and small arrows IVR transitions.

$\tilde{E}_{i \zeta}=E_{i \zeta}+i \Gamma_{\zeta}$, with $\Gamma_{\zeta}$ the damping factor ${ }^{23,24}$ associated with state $\zeta$. Note for conciseness the frequency dependence of the $\alpha_{i j}^{a b}$ factors is now implicit and follows from the superscripts. The dissipative effect of internal vibrational redistribution (IVR) does not itself feature in the electronic interstate kinetics, though the associated redshift in the emitted radiation is necessarily apparent and emerges in the following. For the single-step RET which delivers energy to the acceptor we have the familiar result from second-order timedependent perturbation theory, ${ }^{1}$

$$
\Gamma_{\mathrm{RET}}^{B \leftarrow A}=\frac{2 \pi}{\hbar}\left|\mu_{i}^{0 \alpha(A)} V_{i j}\left(\omega^{\prime}, \mathbf{R}\right) \mu_{j}^{\beta 0(B)}\right|^{2} \boldsymbol{\rho}_{f}^{B} .
$$

Here $\omega^{\prime}$ corresponds to the donor emission frequency, where $\omega^{\prime} \leqslant 2 \omega_{0}$ as illustrated by Fig. $2 ; V_{i j}$ defines the electric dipole-electric dipole coupling tensor, given generally by ${ }^{25}$

$$
\begin{aligned}
V_{i j}\left(\omega_{1}, \mathbf{R}\right)= & \frac{\exp \left(\frac{i \omega_{1} R}{c}\right)}{4 \pi \epsilon_{0} R^{3}}\left\{\left(1-\frac{i \omega_{1} R}{c}\right)\left(\delta_{i j}-3 \hat{R}_{i} \hat{R}_{j}\right)\right. \\
& \left.-\left(\frac{\omega_{1} R}{c}\right)^{2}\left(\delta_{i j}-\hat{R}_{i} \hat{R}_{j}\right)\right\},
\end{aligned}
$$

and the donor-acceptor displacement vector is defined as $\mathbf{R}$ $=\mathbf{R}_{B}-\mathbf{R}_{A}$. As is usual in the study of the systems of interest here, the electric dipole approximation is used in the derivation of Eq. (7) - its legitimacy justified by the focus on strongly allowed transitions in electronically distinct and structurally separated chromophores. Thus the total rate equation for a two-photon resonance energy transfer process emerges as

$$
\begin{aligned}
\left\langle\Gamma^{\mathrm{TPRET}}\right\rangle= & \frac{N_{A} g_{11}^{(2)}}{60}\left(\frac{\langle I\rangle \pi}{\epsilon_{0} c \hbar}\right)^{2}\left\{\left(2|\mathbf{e} \cdot \mathbf{e}|^{2}-1\right) \alpha_{i i}^{\alpha 0(A)} \bar{\alpha}_{j j}^{\alpha 0(A)}\right. \\
& \left.-\left(|\mathbf{e} \cdot \mathbf{e}|^{2}-3\right) \alpha_{i j}^{\alpha 0(A)} \bar{\alpha}_{i j}^{\alpha 0(A)}\right\} \\
& \times\left|\mu_{i}^{0 \alpha(A)} V_{i j}\left(\omega^{\prime}, \mathbf{R}\right) \mu_{j}^{\beta 0(B)}\right|^{2} \rho_{f}^{A^{* *}} \rho_{f}^{B},
\end{aligned}
$$

in which the frequency dependence of the molecular tensors is implicit and $g_{11}^{(2)}$ is the single-site degree of second-order optical coherence, ${ }^{26}$ reflecting the effect of fluctuations in photon count in the laser beam. In detail $g_{11}^{(2)}$ is defined as

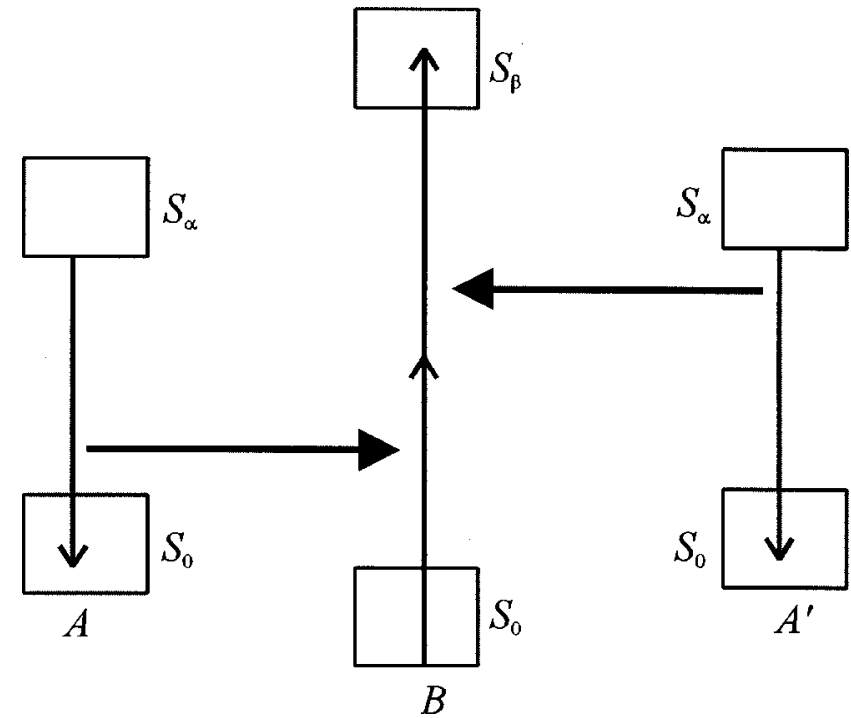

FIG. 3. Energy scheme for cooperative energy pooling: as in Fig. 1, and with $S_{\beta}$ denoting a higher electronic state of the acceptor, $B$ and its associated manifold. Here $A$ and $A^{\prime}$ are interchangeable.

$$
\begin{aligned}
g_{11}^{(2)} & \equiv g^{(2)}\left(\mathbf{R}_{A}, \mathbf{R}_{A} ; \mathbf{R}_{A}, \mathbf{R}_{A}\right) \\
& =\frac{\left\langle\mathbf{d}^{\perp(-)}\left(\mathbf{R}_{A}\right) \mathbf{d}^{\perp(-)}\left(\mathbf{R}_{A}\right) \mathbf{d}^{\perp(+)}\left(\mathbf{R}_{A}\right) \mathbf{d}^{\perp(+)}\left(\mathbf{R}_{A}\right)\right\rangle}{\left\langle\mathbf{d}^{\perp(-)}\left(\mathbf{R}_{A}\right) \mathbf{d}^{\perp(+)}\left(\mathbf{R}_{A}\right)\right\rangle\left\langle\mathbf{d}^{\perp(-)}\left(\mathbf{R}_{A}\right) \mathbf{d}^{\perp(+)}\left(\mathbf{R}_{A}\right)\right\rangle},
\end{aligned}
$$

where the subscript 11 denotes a single-site function and $\mathbf{d}^{\perp}\left(\mathbf{R}_{A}\right)$ is the transverse electric displacement field given by

$$
\begin{aligned}
\mathbf{d}^{\perp}\left(\mathbf{R}_{A}\right)= & \mathbf{i} \sum_{k, \lambda}\left(\frac{\hbar c k \boldsymbol{\epsilon}_{0}}{2 V}\right)^{1 / 2}\left\{\mathbf{e}^{(\lambda)}(\mathbf{k}) a^{(\lambda)}(\mathbf{k}) e^{i\left(\mathbf{k} \cdot \mathbf{R}_{A}\right)}\right. \\
& \left.-\overline{\mathbf{e}}^{(\lambda)}(\mathbf{k}) a^{\dagger\langle\lambda\rangle}(\mathbf{k}) e^{-i\left(\mathbf{k} \cdot \mathbf{R}_{A}\right)}\right\} \\
= & \mathbf{d}^{\perp(+)}\left(\mathbf{R}_{A}\right)+\mathbf{d}^{\perp(-)}\left(\mathbf{R}_{A}\right) .
\end{aligned}
$$

Here $\mathbf{e}^{(\lambda)}(\mathbf{k})$ is the polarization unit vector $\left[\overline{\mathbf{e}}^{(\lambda)}(\mathbf{k})\right.$ being its complex conjugate] and $a^{(\lambda)}(\mathbf{k}), a^{\dagger(\lambda)}(\mathbf{k})$ are respectively the photon annihilation and creation operators for a photon mode $(\mathbf{k}, \lambda)$ (wave vector $\mathbf{k}$ and polarization $\lambda$ ). It is worth noting that the second-order coherence factor, $g_{11}^{(2)}$ is equal to 1 in a fully coherent light field, whereas in a unidirectional light beam of Lorentzian or Gaussian frequency distribution, $g_{11}^{(2)}=2$. Equation (8) will serve as a basis for judging the relative efficiency of two-photon and energy pooling energy transfer mechanisms; the latter is the subject of the following section.

\section{TWIN-DONOR ENERGY POOLING PROCESSES}

In contrast to optically linear light harvesting, ${ }^{19}$ twindonor energy pooling comprises two submechanisms. ${ }^{27}$ These are defined as (a) the cooperative mechanism, where the initial one-photon excitations are followed by RET from both donors directly to the acceptor, and (b) the accretive mechanism, where the initial excitation energy of one donor is passed to its partner and the sum of the two excitations is transferred to the acceptor. These two submechanisms are illustrated by the energy schemes of Figs. 3 and 4. To con- 


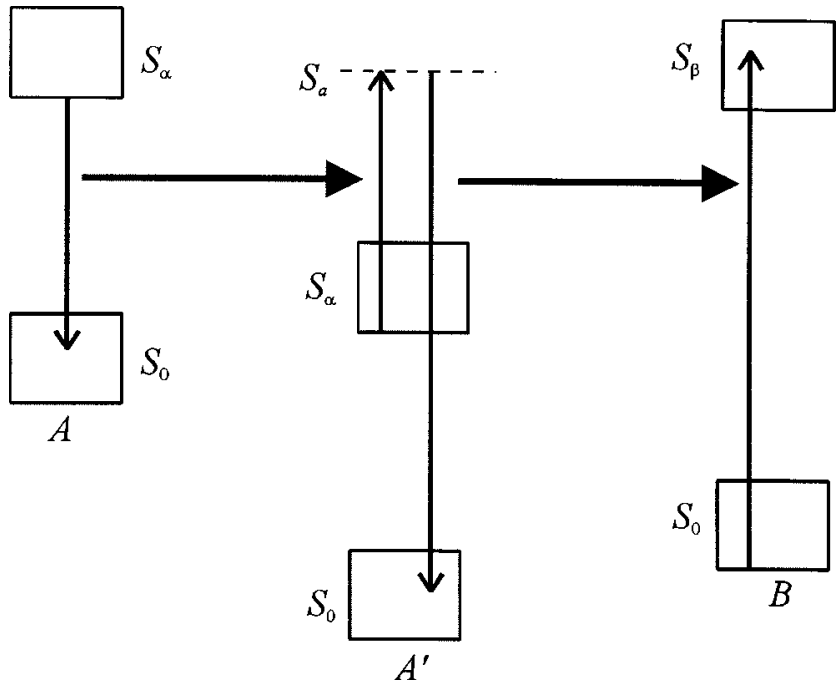

FIG. 4. Energy scheme for accretive energy pooling: $S_{\alpha}$ represents a virtual state.

struct the equation for the overall rate of a pooling process, accommodating both submechanisms, a similar development to that used in the last section is employed, i.e., determining factors include the number of donors contained within the laser focal volume, the probability of two such donors being simultaneously excited, and the rate of twin-donor energy transfer to the acceptor.

First consider the probability of satisfying the initial conditions for excitation of a donor pair. The number of pairs at the laser focus, under standard conditions, is taken to be $1 / 2 N_{A}\left(N_{A}-1\right)$, and if $N_{1 A}$ signifies the number of donors in the relevant electronic excited state, the probability of both partners in any one pair being excited is the square of $N_{1 A} / N_{A}$, assuming the decay lifetime is short compared to the laser pulse duration. Under such steady-state conditions the latter factor is given by

$$
\frac{N_{1 A}}{N_{A}} \approx \frac{k_{A}^{1 \leftarrow 0}}{k_{A}^{0 \leftarrow 1}},
$$

with $k_{A}^{1 \leftarrow 0}$ and $k_{A}^{0 \leftarrow 1}$ representing the one-photon absorption and deexcitation rate constants, respectively. The latter are defined similarly to Eq. (2) and the former are again derived from molecular QED as follows:

$$
k_{A}^{1 \leftarrow 0}=\frac{\pi I}{3 \hbar c \epsilon_{0}}\left|\boldsymbol{\mu}^{\alpha 0(A)}\right|^{2} \rho_{f}^{A^{*}},
$$

where $\boldsymbol{\mu}^{\alpha 0}$ is the transition electric dipole moment connecting the donor ground state and excited state. Inserting Eq. (12) into Eq. (11),

$$
\frac{N_{1 A}}{N_{A}}=\frac{\pi I}{3 \hbar c \epsilon_{0} k_{A}^{0 \leftarrow 1}}\left|\boldsymbol{\mu}^{\alpha 0(A)}\right|^{2} \rho_{f}^{A^{*}} .
$$

Turning to the RET step, we have to consider both energy pooling submechanisms. As shown in earlier work ${ }^{15,27}$ the results emerge from fourth-order time-dependent perturbation theory in the form of the following rate equation:

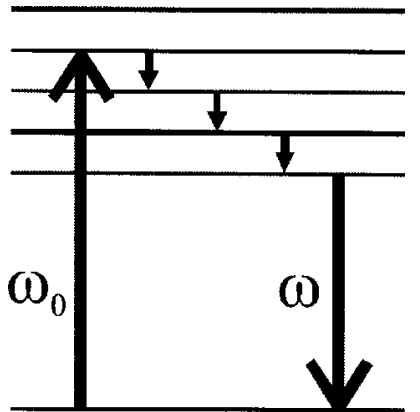

FIG. 5. Nonequivalence of the photon emission frequency $\omega$ and absorption frequency $\omega_{0}$ due to IVR.

$$
\begin{aligned}
\Gamma_{\mathrm{RET}}^{B \leftarrow A, A^{\prime}}= & \frac{2 \pi}{\hbar} \mid \mu_{i}^{0 \alpha(A)} V_{i j}(\omega, \mathbf{R}) \alpha_{j k}^{\beta 0(B)}(-\omega,-\omega) \\
& \times V_{k l}\left(\omega, \mathbf{R}^{\prime}\right) \mu_{l}^{0 \alpha\left(A^{\prime}\right)}+\mu_{i}^{0 \alpha(A)} V_{i j}\left(\omega, \mathbf{R}^{\prime \prime}\right) \\
& \times \alpha_{j k}^{0 \alpha\left(A^{\prime}\right)}\left(\omega^{\prime},-\omega\right) V_{k l}\left(\omega^{\prime}, \mathbf{R}^{\prime}\right) \mu_{l}^{\beta 0(B)} \\
& +\mu_{i}^{0 \alpha\left(A^{\prime}\right)} V_{i j}\left(\omega, \mathbf{R}^{\prime \prime}\right) \alpha_{j k}^{0 \alpha(A)}\left(\omega^{\prime},-\omega\right) \\
& \times\left. V_{k l}\left(\omega^{\prime}, \mathbf{R}\right) \mu_{l}^{\beta 0(B)}\right|^{2} \rho_{f}^{B} .
\end{aligned}
$$

Here the first term corresponds to the cooperative submechanism, while the further two terms relate to accretive transfer. Also $\omega$ corresponds to the donor emission frequency, where $\omega \leqslant \omega_{0}$ due to excited state IVR (as illustrated by Fig. 5) and the displacement vectors are defined as $\mathbf{R}^{\prime}=\mathbf{R}_{B}-\mathbf{R}_{A^{\prime}}, \mathbf{R}^{\prime \prime}$ $=\mathbf{R}_{A^{\prime}}-\mathbf{R}_{A}$.

Putting together the various factors delineated above, the ensemble averaged rate equation for the energy pooling process is as follows:

$$
\begin{aligned}
\left\langle\Gamma^{\text {Pooling }}\right\rangle= & N_{A}\left(N_{A}-1\right)\left(\frac{\pi^{3 / 2}}{3 \hbar^{3 / 2} c \epsilon_{0} k_{A}^{0 \leftarrow 1}}\right)^{2} \\
& \times\langle I\rangle^{2}\left|\boldsymbol{\mu}^{\alpha 0(A)}\right|^{4}\left(\rho_{f}^{A^{*}}\right)^{2} \rho_{f}^{B} \mid \mu_{i}^{0 \alpha(A)} \\
& \times V_{i j}(\omega, \mathbf{R}) \alpha_{j k}^{\beta 0(B)} V_{k l}\left(\omega, \mathbf{R}^{\prime}\right) \mu_{l}^{0 \alpha\left(A^{\prime}\right)} \\
& +\mu_{i}^{0 \alpha(A)} V_{i j}\left(\omega, \mathbf{R}^{\prime \prime}\right) \alpha_{j k}^{0 \alpha\left(A^{\prime}\right)} V_{k l}\left(\omega^{\prime}, \mathbf{R}^{\prime}\right) \mu_{l}^{\beta 0(B)} \\
& +\left.\mu_{i}^{0 \alpha\left(A^{\prime}\right)} V_{i j}\left(\omega, \mathbf{R}^{\prime \prime}\right) \alpha_{j k}^{0 \alpha(A)} V_{k l}\left(\omega^{\prime}, \mathbf{R}\right) \mu_{l}^{\beta 0(B)}\right|^{2} .
\end{aligned}
$$

The excitation dynamics of the chemically equivalent $A$ and $A^{\prime}$ are for simplicity taken to be identical in the initial conditions, but the decay processes are necessarily differentiated in the RET step to accommodate potentially differing orientations in space. Note that, unlike Eq. (8), the above equation does not exhibit a laser coherence factor since energy pooling does not require simultaneous absorption of pump photons by the two donors-and averaged over the excited state lifetime the coherence of the pump radiation loses relevance. In contrast, the TPRET case does require arrival of both photons at (essentially) the same time and hence a dependence on photon statistics emerges in $g_{11}^{(2)}$. 
TABLE I. Transition tensors and their corresponding irreducible representations for both donor and acceptor species in each of the three mechanisms.

\begin{tabular}{lcccc}
\hline \hline Mechanism & Transition & $\begin{array}{c}\text { IR(s) of donor } \\
\text { chromophores }\end{array}$ & Transition & $\begin{array}{c}\text { IR(s) of } \\
\text { acceptor } \\
\text { chromophores }\end{array}$ \\
\hline TPRET & $\begin{array}{c}\boldsymbol{\alpha}^{\alpha 0(A)} \\
\boldsymbol{\mu}^{0 \alpha(A)}\end{array}$ & $\begin{array}{c}D^{(0+)} \oplus D^{(2+)} \\
D^{(1-)}\end{array}$ & $\boldsymbol{\mu}^{\beta 0(B)}$ & $D^{(1-)}$ \\
Cooperative & $\begin{array}{c}\boldsymbol{\mu}^{\alpha 0(A)}, \boldsymbol{\mu}^{0 \alpha(A)} \\
\boldsymbol{\mu}^{\alpha 0\left(A^{\prime}\right)}, \boldsymbol{\mu}^{0 \alpha\left(A^{\prime}\right)}\end{array}$ & $D^{(1-)}$ & $\boldsymbol{\alpha}^{\beta 0(B)}$ & $D^{(0+)} \oplus D^{(2+)}$ \\
Accretive & $\boldsymbol{\mu}^{\alpha 0(A)}, \boldsymbol{\mu}^{0 \alpha(A)}$, & $\boldsymbol{\mu}^{\beta 0(B)}$ & $D^{(1-)}$ \\
& $\boldsymbol{\mu}^{\alpha 0\left(A^{\prime}\right)}$ & $D^{(1-)}$ & & \\
\hline \hline
\end{tabular}

\section{RELATIVE EFFICIENCIES}

The starting point for an exploration of the structural and electronic design factors, and their bearing on the relative efficiencies of each mechanism, we take the ratio of Eqs. (8) and (15), to produce the result

$$
\begin{aligned}
\frac{\left\langle\Gamma^{\text {Pooling }}\right\rangle}{\left\langle\Gamma^{\mathrm{TPRET}}\right\rangle}= & \frac{20 \pi\left(N_{1 A}-1\right)\left(\rho_{f}^{A^{*}}\right)^{2}}{3 \hbar g_{11}^{(2)} \rho_{f}^{A^{* *}}}\left(\frac{k_{A}^{0 \leftarrow 2}}{\left(k_{A}^{0 \leftarrow 1}\right)^{2}}\right)\left(\frac{\left|\boldsymbol{\mu}^{\alpha 0(A)}\right|^{4}}{\left(2|\mathbf{e} \cdot \mathbf{e}|^{2}-1\right) \alpha_{i i}^{\alpha 0(A)} \bar{\alpha}_{j j}^{\alpha 0(A)}-\left(|\mathbf{e} \cdot \mathbf{e}|^{2}-3\right) \alpha_{i j}^{\alpha 0(A)} \bar{\alpha}_{i j}^{\alpha 0(A)}}\right) \\
& \times\left(\frac{\left|\mu_{i}^{0 \alpha(A)} V_{i j}(\omega, \mathbf{R}) \alpha_{j k}^{\beta 0(B)} V_{k l}\left(\omega, \mathbf{R}^{\prime}\right) \mu_{l}^{0 \alpha\left(A^{*}\right)}+\left[\mu_{l}^{0 \alpha(A)} V_{i j}\left(\omega, \mathbf{R}^{\prime \prime}\right) \alpha_{j k}^{0 \alpha(A)} V_{k l}\left(\omega^{\prime}, \mathbf{R}^{\prime}\right) \mu_{l}^{\beta 0(B)}+A \leftrightarrow A^{\prime}\right]^{1}\right|^{2}}{\left|\mu_{i}^{0 \alpha(A)} V_{i j}\left(\omega^{\prime}, \mathbf{R}\right) \mu_{j}^{\beta 0(B)}\right|^{2}}\right),
\end{aligned}
$$

where $A \leftrightarrow A^{\prime}$ specifies a term with $A$ and $A^{\prime}$ interchanged in comparison to the previous term. In the following, attention focuses on a number of key facets of the above result. These are optical selection rules, exciton effects, spectral overlap, quantum interference, and the nanoscale architecture.

\section{A. Optical selection rules}

Each of the mechanisms for optically nonlinear photoactivity entails a different form of interaction for the donor and acceptor units. The selection rules for one-photon and twophoton processes differ and, for a given system, one or more of the mechanisms or submechanisms may be forbidden by local symmetry-noting, however, that the local electronic environment and the quasicontinuum form of vibrational sublevels are factors that can to some extent modify the selection rules. The transition dipole moments $\boldsymbol{\mu}^{a b}$ for singlephoton processes are associated with the irreducible representation (irrep) $D^{(1-)}$ of an odd-parity rank 1 tensor, while for the two-photon interactions the representations of the even-parity rank 2 tensors $\boldsymbol{\alpha}^{a b}$ comprise the irreps $D^{(0+)}$ $\oplus D^{(1+)} \oplus D^{(2+)} .{ }^{28}$ The $(1+)$ irrep components of the twophoton tensors vanish only if the latter relate to two energetically equivalent photon events (e.g., the absorptions of two photons having identical frequency) — otherwise they remain, as, for example, in any resonance Raman scattering process. The nature of the associated donor and acceptor transitions imposes conditions on the validity of each mechanism. Generally, the direct product of the initial and final state representations must span at least one of the irrep components of the relevant transition tensors. These transition tensors and their corresponding irrep components are shown in Table I for each of the three mechanisms. An example is the accretive submechanism, which is only symmetry-allowed if the donor decay transition has transformation properties replicated by a component of $D^{(1-)}$, i.e., the irrep for the initial photoabsorption. Furthermore, the same transition must have the transformation properties associated with a component of $D^{(0+)} \oplus D^{(1+)} \oplus D^{(2+)}$. The excitation transition dipole moment of the acceptor will also need to transform as one or more components of $D^{(1-)}$.

The detailed form of the irrep components is governed by the local point group symmetry $D_{3 h}, D_{4 h}$, and $C_{2 v}$ in many dendrimeric materials. According to the chromophore architecture, specific conclusions can be drawn for each of two main classes of dendrimeric light-harvesting materials, as follows:

$D_{3 h}$ systems. Examples of systems of $D_{3 h}$ symmetry for both acceptor and donor chromophores are materials based on polyphenylethynl ${ }^{29,30}$ dendrimers, which have been the subject of considerable development following pioneering work by Xu and Moore. ${ }^{31}$ Here the detailed form of the irrep components for the $D_{3 h}$ case, illustrated by Table II, reveals that when the direct product of the donor ground and excited state representations includes the irrep $E^{\prime}$, all of the discussed mechanisms are permitted-whereas only cooperative pooling is allowed when the same product spans $A_{2}^{\prime \prime}$. (Note the assumption of equivalence between the absorption and emission transition symmetries of the donor species.) 
TABLE II. Irrep and components for both donor and acceptor species for the three mechanisms, where both species are of $D_{3 h}$ symmetry. SE denotes symmetry element.

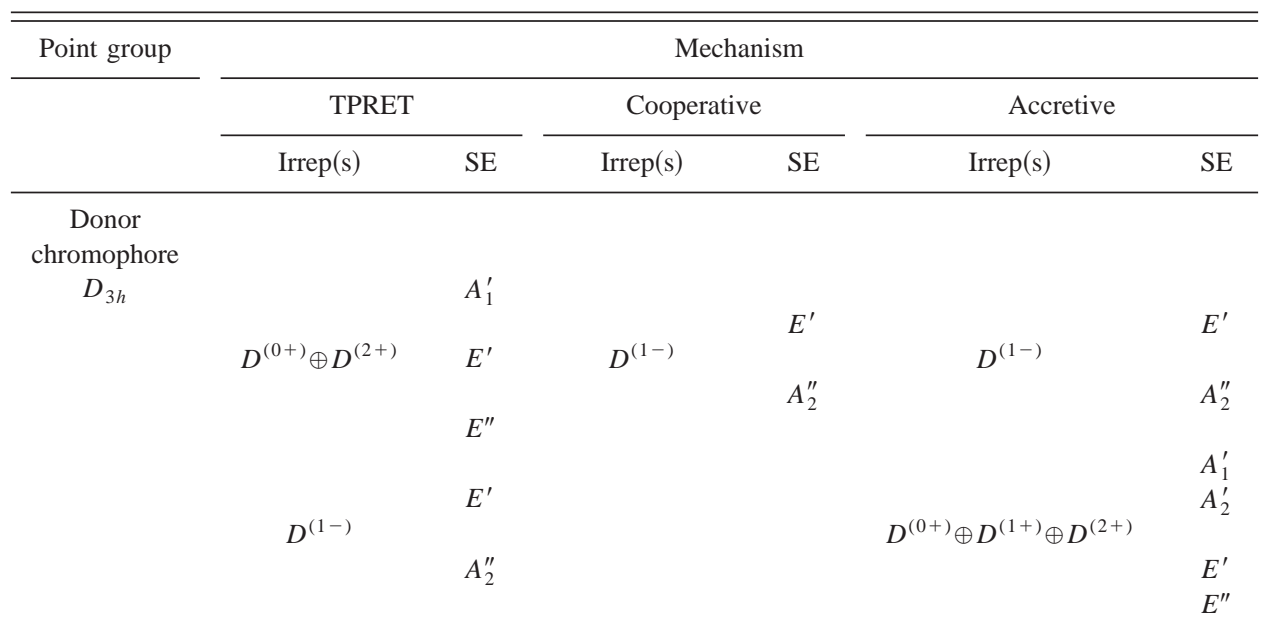

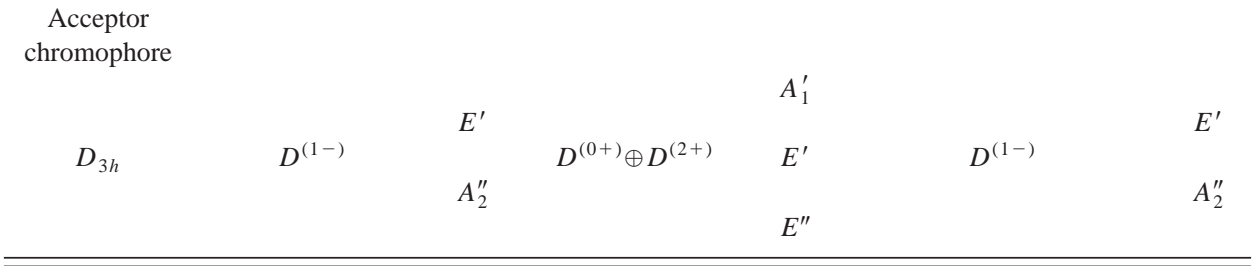

$C_{2 v} / D_{4 h}$ systems. Examples of systems based on $C_{2 v}$ and $D_{4 h}$ symmetry for the donor and acceptor species respectively are light-harvesting assemblies of porphyrin $^{5,6,32-38}$ and multiporphyrin arrays ${ }^{6,39,40}$ —energy transfer in the latter is especially efficient from Zn-containing porphyrin to free-base porphyrin. ${ }^{41}$ The irrep components for $C_{2 v}$ and $D_{4 h}$ symmetry are given by Table III and show, in the donor species case, that all three mechanisms are symmetry allowed through transitions of $A_{1}, B_{1}$, and $B_{2}$ symmetry; in addition, both TPRET and accretive processes are allowed through $A_{2}$ transitions. For the acceptor, transitions with $A_{2 u}$ and $E_{u}$ symmetry permit the TPRET and accretive mechanisms, while $A_{1 g}, B_{1 g}, B_{2 g}$, and $E_{g}$ allow cooperative transfer.

\section{B. Exciton effects}

In general, the initiation of TPRET requires only one excited donor and the initial energy deposition is localized to that species. Often, dendrimeric materials are designed with spacer units separating the donors and acceptors, so as to retain their distinct electronic integrity and preclude charge transfer. However, if two or more identical donor species within the proximity of each other are electronically coupled to any significant extent, an exciton may form. Excitons in this context $\mathrm{t}^{42}$ are associated with an uncertainty in the location of the photon energy deposition and are generated when the number of excitations within a chromophore array is less than the number of donors it comprises. Hence energy pooling processes, which require two excited donor species, may engage three or more donors in excitonic states. Consider, for example, a threefold symmetric, nodal component of a dendrimer comprising chemically identical donors $A, B$, and $C$ each at one corner of an equilateral triangle, with an accep- tor, $D$, at the center. Both in TPRET and energy pooling a donor exciton intermediate can form, as is illustrated by the equation,

$$
\begin{gathered}
A+B+C+D+2 \hbar \omega \stackrel{\text { Exciton formation }}{\longrightarrow}(A+B+C)^{* *}+D \\
\stackrel{\text { RET }}{\longrightarrow} A+B+C+D^{*} .
\end{gathered}
$$

Although associated in each case with the energy of two input photons, the exciton is recognized to have a different structure for the two processes. Specifically, $(A+B+C)^{* *}$ is one of two forms-either $\left(A^{* *}+B+C\right)$ and its permutations for TPRET, or $\left(A^{*}+B^{*}+C\right)$ and permutations for energy pooling-each form shown explicitly in terms of wave functions below. ${ }^{43,44}$ For TPRET, in general, the exciton state $\left|\Psi_{j}^{\prime \prime}\right\rangle$ is given by

$$
\begin{aligned}
\left|\Psi_{j}^{\prime \prime}\right\rangle= & \frac{1}{\sqrt{3}}\left(\left|A^{u^{\prime}} B^{0} C^{0} D^{0}\right\rangle+c^{ \pm}\left|A^{0} B^{u^{\prime}} C^{0} D^{0}\right\rangle\right. \\
& \left.+c^{\mp}\left|A^{0} B^{0} C^{u^{\prime}} D^{0}\right\rangle\right) .
\end{aligned}
$$

Here the superscript $u^{\prime}$ indicates the two-photon excited state of the pertinent donor species and the coefficients $c^{ \pm}$ and $c^{\overline{ }}$ are introduced as a means to generate each exciton state, written explicitly these are

$$
c^{ \pm}=1+c^{\prime}\left(-\frac{3}{2} \pm i \frac{\sqrt{3}}{2}\right),
$$

putting $c^{\prime}=0$ gives the $\left|\Psi_{1}\right\rangle$ state; with $c^{\prime}=1$ the upper sign in Eq. (18) yield $\left|\Psi_{2}\right\rangle$ and the lower $\left|\Psi_{3}\right\rangle$. The corresponding two-photon exciton state energy, $E_{l}^{\prime \prime}$, is taken to be

$$
E_{1}^{\prime \prime}=E^{\prime}+2 v^{\prime}, \quad E_{2}^{\prime \prime}=E_{3}^{\prime \prime}=E^{\prime}-v^{\prime},
$$


TABLE III. Irrep and components for both donor and acceptor species for the three mechanisms, where the donor is of $C_{2 v}$ symmetry and the acceptor is $D_{4 h}$. SE denotes symmetry element.

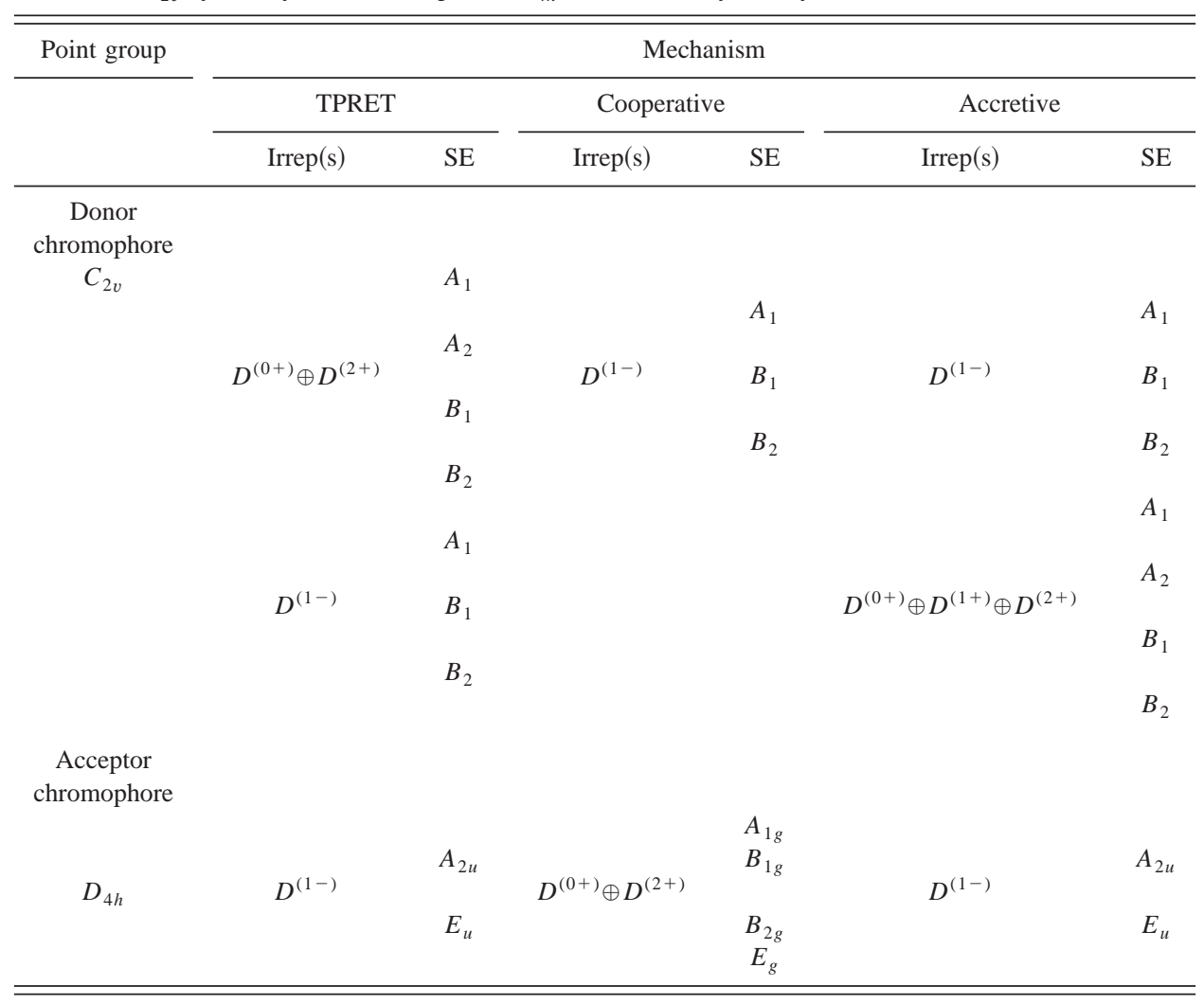

which incorporates $E^{\prime}=\left\langle\xi^{u^{\prime}}\left|H_{\xi}\right| \xi^{u^{\prime}}\right\rangle$ and $v^{\prime}$ $=\left\langle\xi^{u^{\prime}}\left|V_{\xi \xi^{\prime}}\right| \xi^{u^{\prime}}\right\rangle$, where $H_{\xi}$ denotes the Hamiltonian for donor $\xi$ and $V_{\xi \xi^{\prime}}$ is the interaction between species $\xi$ and $\xi^{\prime}$. For energy pooling, in the general form, the single-photon exciton state $\left|\Psi_{j}^{\prime}\right\rangle$ is given by

$$
\begin{aligned}
\left|\Psi_{j}^{\prime}\right\rangle= & \frac{1}{\sqrt{3}}\left(\left|A^{u} B^{u} C^{0} D^{0}\right\rangle+c^{ \pm}\left|A^{u} B^{0} C^{u} D^{0}\right\rangle\right. \\
& \left.+c^{\mp}\left|A^{0} B^{u} C^{u} D^{0}\right\rangle\right) .
\end{aligned}
$$

The superscript $u$ indicates a one-photon excited species and we have one ground-state and two excited-state donor species instead of vice versa. Thus $E=\left\langle\xi^{u}\left|H_{\xi}\right| \xi^{u}\right\rangle, \quad v$ $=\left\langle\xi^{u}\left|V_{\xi \xi^{\prime}}\right| \xi^{\prime} u\right.$, and $\left.\mid \Psi_{1}^{\prime}\right\rangle,\left|\Psi_{2}^{\prime}\right\rangle,\left|\Psi_{3}^{\prime}\right\rangle$ are generated from Eq. (20) using Eq. (18) with the exciton state energy, $E_{j}^{\prime}$ as follows:

$$
E_{1}^{\prime}=2 E+2 v, \quad E_{2}^{\prime}=E_{3}^{\prime}=2 E-v .
$$

Equation (21) exhibits a Davydov splitting between $E_{1}^{\prime}$ and the degenerate $E_{2}^{\prime}$ and $E_{3}^{\prime}$ as seen previously in Eq. (19). As the number of donors surrounding an acceptor on a given dendrimer node increases, the significance of excitonic transfer also grows, and increasingly favors the accretive mechanism-a point we return to in Sec. IV E below.

\section{Spectral overlap}

The detailed form of spectral overlap associated with each mechanism is a matter of considerable interest. As in conventional single-donor energy transfer, to determine a rate equation connected to Förster theory for TPRET mechanisms requires the consideration of spectral overlap between the donor fluorescence spectrum and the dispersive absorption cross section of the acceptor-the detailed form depending on distance, and in the short range leading to the familiar Förster result. The QED formulation of this rate equation is given by ${ }^{45}$

$$
\Gamma_{\mathrm{RET}}^{B \leftarrow A}=\frac{9}{8 \pi c^{2} \tau_{A}} \int_{0}^{\infty} F_{A}\left(\omega^{\prime}\right) \sigma_{B}\left(\omega^{\prime}\right) \omega^{\prime 2} g\left(\omega^{\prime}, \mathbf{R}\right) d \omega^{\prime} .
$$

Equation (22) includes the radiative lifetime of the donor, $\tau_{A}$, the cross section of the acceptor absorption and donor emission spectra, these are defined generally as

$$
\begin{aligned}
\sigma_{B}(\omega)= & \frac{\pi \omega}{3 \epsilon_{0} c}\left|\boldsymbol{\mu}^{0 \beta(B)}\right|^{2} \sum_{m, p} \rho_{B}^{(m)}\left|\left\langle\varphi_{B}^{(p)} \mid \varphi_{B}^{(m)}\right\rangle\right|^{2} \\
& \times \delta\left(e_{B_{p}^{*}}-e_{B_{m}}-\hbar \omega\right),
\end{aligned}
$$

and

$$
\begin{aligned}
F_{A}(\omega)= & \frac{\omega^{3} \tau_{A}}{3 \epsilon_{0} \pi \hbar c^{3}}\left|\boldsymbol{\mu}^{\alpha 0(A)}\right|^{2} \sum_{n, r} \rho_{A *}^{(n)}\left|\left\langle\varphi_{A}^{(r)} \mid \varphi_{A *}^{(n)}\right\rangle\right|^{2} \\
& \times \delta\left(e_{A_{n}^{*}}-e_{A_{r}}-\hbar \omega\right),
\end{aligned}
$$

respectively. Here, $\rho$ is the population distribution function of the initial vibrational states of the specified species, each of the indices $n, r, m, p$ specifies the set of vibrational, etc. sublevels of the transfer species, the energies of the initial 
and final state of each species are included in the energyconserving $\delta$ function. In detail $F_{A}(\omega)$ is determined by the exit state of $A$, which is a consequence of initial excitation and subsequent IVR. Also included in Eq. (22) is $g\left(\omega^{\prime}, \mathbf{R}\right)$ which emerges from Eq. (7) and is generally given by

$$
g(\omega, \mathbf{R})=\eta_{3}^{2} \frac{c^{6}}{\omega^{6} R^{6}}+\left(\eta_{3}^{2}-2 \eta_{1} \eta_{3}\right) \frac{c^{4}}{\omega^{4} R^{4}}+\eta_{1}^{2} \frac{c^{2}}{\omega^{2} R^{2}},
$$

where $\eta_{q}$ are the orientational factors and written, in general, as

$$
\eta_{q}=\left(\hat{\boldsymbol{\mu}}_{A} \cdot \hat{\boldsymbol{\mu}}_{B}\right)-q\left(\hat{\mathbf{R}} \cdot \hat{\boldsymbol{\mu}}_{A}\right)\left(\hat{\mathbf{R}} \cdot \hat{\boldsymbol{\mu}}_{B}\right) \quad(q=1,3) .
$$

The leading term of Eq. (25) carries an orientational dependence for which $q=3$, as befits the usual form of dipoledipole coupling, whereas in the long range the form with $q$ $=1$ dominates-as has been shown in previous work. ${ }^{45}$ In the short-range Eq. (22) takes the form of the Förster rate, which is given as follows:

$$
\Gamma_{\mathrm{RET}}^{B \leftarrow A}=\frac{9 c^{4} \eta_{3}^{2}}{8 \pi \tau_{A} R^{6}} \int_{0}^{\infty} F_{A}\left(\omega^{\prime}\right) \sigma_{B}\left(\omega^{\prime}\right) \omega^{\prime-4} d \omega^{\prime} .
$$

The form of spectral overlap associated with the energy pooling processes, i.e., twin-donor transfer, is more complex due to the energy transferal of $\hbar \omega$ from two donor species to an acceptor. The rate observable accommodates both submechanisms and also their quantum interference (see Sec. IV D) and is given by the following: ${ }^{46}$

$$
\begin{aligned}
\Gamma_{\mathrm{RET}}^{B \leftarrow A, A^{\prime}}= & \frac{2 \pi \rho_{f}^{B}}{\hbar}\left\{\left|M_{\mathrm{fi}}^{\mathrm{acc} 1}\left(\mathbf{R}^{\prime}, \mathbf{R}^{\prime \prime}\right)\right|^{2}+\left|M_{\mathrm{fi}}^{\mathrm{acc} 2}\left(\mathbf{R}, \mathbf{R}^{\prime \prime}\right)\right|^{2}\right. \\
& +\left|M_{\mathrm{fi}}^{\mathrm{coop}}\left(\mathbf{R}, \mathbf{R}^{\prime}\right)\right|^{2} \\
& +2 \operatorname{Re}\left[M_{\mathrm{fi}}^{\mathrm{acc} 1}\left(\mathbf{R}^{\prime}, \mathbf{R}^{\prime \prime}\right) \bar{M}_{\mathrm{fi}}^{\mathrm{acc} 2}\left(\mathbf{R}, \mathbf{R}^{\prime \prime}\right)\right. \\
& +M_{\mathrm{fi}}^{\mathrm{acc1}}\left(\mathbf{R}^{\prime}, \mathbf{R}^{\prime \prime}\right) M_{\mathrm{fi}}^{\mathrm{coop}}\left(\mathbf{R}, \mathbf{R}^{\prime}\right) \\
& \left.\left.+M_{\mathrm{fi}}^{\mathrm{acc} 2}\left(\mathbf{R}, \mathbf{R}^{\prime \prime}\right) M_{\mathrm{fi}}^{\mathrm{coop}}\left(\mathbf{R}, \mathbf{R}^{\prime}\right)\right]\right\},
\end{aligned}
$$

where $M_{\mathrm{fi}}$ is the quantum amplitude of cooperative (coop) or accretive $($ acc1,acc2) transfer corresponding to the relevant terms within the modulus in Eq. (14)—acc1, acc2 differentiate contributions which differ only through the interchange of $A$ and $A^{\prime}$. For example, the cooperative component of Eq. (28) (which also signifies the rate expression for a system in which selection rules preclude accretive transfer) is given by

$$
\begin{aligned}
\left|M_{\mathrm{fi}}^{\mathrm{coop}}\left(\mathbf{R}, \mathbf{R}^{\prime}\right)\right|^{2}= & \frac{9}{64 \pi^{2} c^{4} \tau_{A} \tau_{A^{\prime}}} \iint \omega^{2}\left(\omega^{\prime}-\omega\right)^{2} \\
& \times F_{A}(\omega) F_{A^{\prime}}\left(\omega^{\prime}-\omega\right) \tilde{\sigma}_{B}\left(\omega, \omega^{\prime}-\omega\right) \\
& \times g(\omega, \mathbf{R}) g\left(\omega^{\prime}-\omega, \mathbf{R}^{\prime}\right) d \omega d \omega^{\prime},
\end{aligned}
$$

where $F_{A}$ and $F_{A^{\prime}}$ are both given by Eq. $(24), g(\omega, \mathbf{R})$ by Eq. (25) and the cross section of the acceptor two-photon absorption, $\widetilde{\sigma}_{B}\left(\omega, \omega^{\prime}-\omega\right)$, is determined by writing the rate of two-photon absorption as follows:

$$
\begin{aligned}
\Gamma_{\mathrm{TPA}}= & \frac{\pi h n n^{\prime} \omega\left(\omega^{\prime}-\omega\right)}{2 \epsilon_{0}^{2} V^{2}}\left|\boldsymbol{\alpha}^{B}\left(\omega, \omega^{\prime}-\omega\right)\right|^{2} \\
& \times \sum_{m, p} \rho_{B}^{(m)}\left|\left\langle\varphi_{B^{*}}^{(p)} \mid \varphi_{B}^{(m)}\right\rangle\right|^{2} \delta\left(e_{B_{p}^{*}}-e_{B_{m}}-\hbar \omega^{\prime}\right) \\
= & \frac{\widetilde{\sigma}_{B}\left(\omega, \omega^{\prime}-\omega\right) n n^{\prime} c^{2}}{V^{2}} .
\end{aligned}
$$

Here $n$ and $n^{\prime}$ are photon numbers derived from number states and $V$ is the quantization volume. Equation (30) can be rearranged to give

$$
\begin{aligned}
\tilde{\sigma}_{B}\left(\omega, \omega^{\prime}-\omega\right)= & \frac{\pi \hbar \omega\left(\omega^{\prime}-\omega\right)}{2 \epsilon_{0}^{2} c^{2}}\left|\boldsymbol{\alpha}^{B}\left(\omega, \omega^{\prime}-\omega\right)\right|^{2} \\
& \times \sum_{m, p} \rho_{B}^{(m)}\left|\left\langle\varphi_{B}^{(p)} \mid \varphi_{B}^{(m)}\right\rangle\right|^{2} \\
& \times \delta\left(e_{B_{p}^{*}}-e_{B_{m}}-\hbar \omega^{\prime}\right) .
\end{aligned}
$$

For the case where selection rules dictate that only accretive transfer occurs, three terms persist (acc1, acc2, and their interference) and the appropriate result can again be directly recovered from Eq. (28).

\section{Quantum interference}

The processes of energy pooling and TPRET lead from the same initial state to a final state in which the acceptor is electronically excited. However the dissipation of energy through IVR in the donor ensemble is different in the two cases so that, whereas these mechanisms may compete if both are allowed, they cannot display quantum interference. That is not the case, however, when we consider the two submechanisms for energy pooling, because the two electronic couplings that each of these involves are concerted and not step-wise processes, as the QED calculations show. In other words the quantum pathways from the initial to the final state traverse only virtual states, in which energy losses are not sustained into or beyond the femtosecond timescale. In the numerator of the last factor in Eq. (16) the cooperative submechanism is represented by the first term and accretive transfer by the subsequent terms. In multiplying the result by its complex conjugate it emerges that there is a cross-term representing the quantum interference of the two submechanisms, which is of clear physical significance and entails interdependent chromophore separation vectors. The cooperative and accretive pathways from the initial to final state of the system, along with the quantum interference of these pathways are schematically illustrated by Fig. 6. In the unfolding technology of dendrimeric and other related nanomaterials, the significance of such quantum interferences should not be underestimated.

\section{E. Nanoscale architecture}

For a given overall geometry, the detailed nanoscale architecture also has a considerable effect on the dominance of one energy pooling submechanism over the other. ${ }^{47}$ To pursue a readily comprehensible physical interpretation we can 


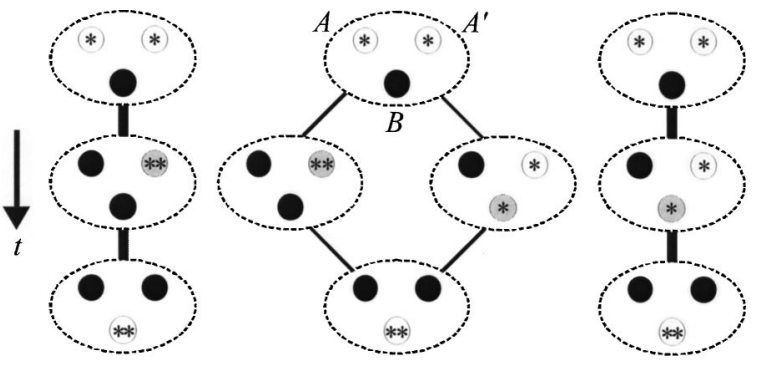

FIG. 6. Energy pooling pathways: accretive and cooperative pathways are left and right, respectively, center depicts the quantum interference of these two pathways. White, blank, and shaded circles denote excited, ground and virtual states species, respectively, asterisks represent the locale of excitation(s), and thick connecting lines signify single channel contributions; thin lines denote interfering channels. Note $A$ and $A^{\prime}$ are interchangeable and time, $t$, flows down the diagram.

first entertain the gross assumption that all transition dipole moments and separation vectors are equivalent, and that components of each of the $\alpha$ tensors have similar magnitude to the corresponding polarizability components. Then, it is possible to write the intimidating expression within the final bracket of Eq. (16) in the greatly simplified form that follows:

$$
\frac{\Gamma^{\text {Pooling }}}{\Gamma^{\text {TPRET }}} \sim \frac{54 \alpha^{\prime 2}}{R^{6}},
$$

where $\alpha^{\prime}$ is a volume polarizability. Equation (32) shows that light-harvesting systems based on small, essentially nonpolarizable chromophores with tightly bound electrons (small $\alpha^{\prime}$ ) are generally dominated by TPRET, while energy pooling is favored by systems with tightly packed donors and acceptors (small separations). In the latter case specifically, cooperative transfer is favored for systems with a highly polarizable acceptor, accretive transfer for those with highly polarizable donors.

The chromophore architecture also plays a role in determining the preferred energy transfer mechanism. Thus, for energy pooling processes in dendrimeric systems with a threefold symmetric nodal motif, the cooperative submechanism is promoted by the closer proximity of the donor and acceptor species in comparison to the donor-donor separation. This follows from the form of the coupling tensors in the two cases, $V_{i j}(\omega, \mathbf{R}) V_{k l}\left(\omega, \mathbf{R}^{\prime}\right)$ for the cooperative mechanism and $V_{i j}\left(\omega, \mathbf{R}^{\prime \prime}\right) V_{k l}\left(\omega, \mathbf{R}^{\prime}\right)$ for the accretive, bearing in mind that in the short range, each $\mathbf{V}(\omega, \mathbf{R})$ has an overall dependence on $R^{-3}$ [see Eq. (7)], where $R$ is the magnitude of the displacement vector in the argument. As the number of donor chromophores around the acceptor increases the accretive submechanism becomes of increasing importance as shown by the ratios of Table IV.

TABLE IV. Relative importance of the accretive submechanism for energy pooling from a ring of $n$ donors.

\begin{tabular}{cccccccc}
\hline \hline$n$ & 3 & 4 & 5 & 6 & 7 & 8 & 9 \\
\hline acc:coop & 0.037 & 0.125 & 0.379 & 1.000 & 2.34 & 4.97 & 9.76 \\
\hline \hline
\end{tabular}

\section{DISCUSSION}

In this paper we have begun to address the principles associated with a multitude of factors whose interplay determines the favored mechanism for optically nonlinear photoactivity. In general, nanomaterials of this kind are designed to expedite one specific mechanism. Examples of energy pooling materials are given in Refs. 4-7, 29-40, and 48-51; recent examples of TPRET dendrimers are given in Refs. $8-12$. Previously, it has not been generally recognized that the two mechanisms can operate in parallel. This work has demonstrated the need to properly accommodate such a possibility, given suitably placed chromophore energy levels, and subject to the geometric and symmetry-based criteria detailed above.

It is interesting to note that TPRET is found in other quite different areas of application. One example is where RET is involved in two-photon three-dimensional imaging ${ }^{52-56}$ - a technique which is especially advantageous for biological specimens due to the enhanced depth profiling and reduced photolytic damage. Also, energy harvesting dendrimers are increasingly being developed for use in organic light-emitting diode materials. ${ }^{57-61}$ Furthermore, energy pooling porphyrin dendrimers have begun to find an application in photodynamic therapy as photosensitizers $^{34,62-64}$ _energy harvesting here leading to the photochemical destruction of cancer cells via generation of singlet oxygen. As results emerge in each of these and other new areas, the relative importance of TPRET and energy pooling as competing processes can also now be examined in the appropriate detailed context, using the results we have reported.

In the present analysis our work has identified considerable intricacies in the theory of nonlinear energy transfer, arising from the multiplicity of time-orderings. Whilst we have restricted consideration to features that arise in the pump-probe configuration, the situation becomes substantially more complex if one allows the possibility of energy transfer occurring whilst pump radiation is still present in the system. Although this will not usually be significant over the timescales associated with ultrashort pulsed laser input, under suitable conditions one can envisage the operation of two further mechanisms, as previously identified by Stockmann. ${ }^{13}$ One such mechanism is two-photon absorption by the acceptor through acquisition of one quantum of energy from a singly excited donor and another from the throughput radiation. The other mechanism is where a singly excited donor is excited to a virtual state by the additional absorption of a photon from the pump radiation field, coupled with resonance energy transfer to the acceptor. In each of these mechanisms a real state is realized after the absorption of one pump photon, rather than two. Although they do not arise under the pump-probe conditions we have assumed, these mechanisms and the complex photodynamics with which they must be associated will undoubtedly prove to be of interest.

Clearly there is considerable scope for the further exploration and exploitation of this diversity of mechanisms. In future work we plan to accommodate and quantify local field corrections to properly reflect the electronic environments of 
the donor and acceptor chromophores. Further, to carry this area of research forward for application to real dendrimeric and allied polymer materials, attention must be paid to the flexibility of each system with regard to its secondary structure and packing. ${ }^{65-67}$ In fact, many high-generation dendrimers are more closely biomimetic precisely because of their essentially globular habit—a facet hidden by the common graphical depictions. ${ }^{68}$ It is our hope that as increasingly detailed principles emerge, these will inform and steer future efforts in the creation of light-harvesting nanomaterials.

\section{ACKNOWLEDGMENTS}

D.S.B. is funded by a studentship from the Engineering and Physical Sciences Research Council. We thank Dr. Luciana Dávila Romero for helpful comments on this work.

${ }^{1}$ Resonance Energy Transfer, edited by D. L. Andrews and A. A. Demidov (Wiley, Chichester, 1999).

${ }^{2}$ P. R. Selvin, Nat. Struct. Biol. 7, 730 (2000).

${ }^{3}$ D. L. Andrews, in Introduction to Complex Mediums for Optics and Electromagnetics, edited by W. S. Weiglhofer and A. Lakhtakia (SPIE, Bellingham, Washington, 2003), pp. 141-163.

${ }^{4}$ A. Archut and F. Vögtle, Chem. Soc. Rev. 27, 233 (1998).

${ }^{5}$ A. Adronov and J. M. J. Fréchet, Chem. Commun. (Cambridge) 2000, 1701.

${ }^{6}$ V. Balzani, P. Ceroni, A. Juris, M. Venturi, S. Campagna, F. Puntoriero, and S. Serroni, Coord. Chem. Rev. 219-221, 545 (2001).

${ }^{7}$ V. Balzani, P. Ceroni, M. Maestri, and V. Vicinelli, Curr. Opin. Chem. Biol. 7, 657 (2003).

${ }^{8}$ A. Adronov, J. M. J. Fréchet, G. S. He, K.-S. Kim, S.-J. Chung, J. Swiatkiewicz, and P. N. Prasad, Chem. Mater. 12, 2838 (2000).

${ }^{9}$ G. S. He, T.-C. Lin, Y. Cui, P. N. Prasad, D. Brousmiche, J. M. Serin, and J. M. J. Fréchet, Opt. Lett. 28, 768 (2003).

${ }^{10}$ D. Brousmiche, J. M. Serin, J. M. J. Fréchet, G. S. He, T.-C. Lin, S.-J. Chung, and P. N. Prasad, J. Am. Chem. Soc. 125, 1448 (2003).

${ }^{11} \mathrm{O}$. Mongin, J. Brunel, L. Porrès, and M. Blanchard-Desce, Tetrahedron Lett. 44, 2813 (2003).

${ }^{12}$ D. Brousmiche, J. M. Serin, J. M. J. Fréchet et al., J. Phys. Chem. B. 108, 8592 (2004).

${ }^{13}$ M. I. Stockmann, Sov. Phys. JETP 60, 49 (1984).

${ }^{14}$ P. Allcock and D. L. Andrews, J. Chem. Phys. 108, 3089 (1998).

${ }^{15}$ R. D. Jenkins and D. L. Andrews, J. Phys. Chem. A 102, 10834 (1998).

${ }^{16}$ K. Kondo, S. Yasuda, T. Sakaguchi, and M. Miya, J. Chem. Soc., Chem. Commun. 1995, 55.

${ }^{17}$ A. Bar-Haim and J. Klafter, J. Phys. Chem. B 102, 1662 (1998).

${ }^{18}$ S. Tretiak, V. Chernyak, and S. Mukamel, J. Phys. Chem. B 102, 3310 (1998).

${ }^{19}$ A. Bar-Haim and J. Klafter, J. Lumin. 76\&77, 197 (1998).

${ }^{20}$ Y. Wakabayashi, M. Tokeshi, D.-L. Jiang, T. Aida, and T. Kitamori, J. Lumin. 83\&84, 313 (1999)

${ }^{21}$ J. L. Bentz, F. N. Hosseini, and J. J. Kozak, Chem. Phys. Lett. 370, 319 (2003).

${ }^{22}$ D. P. Craig and T. Thirunamachandran, Molecular Quantum Electrodynamics (Dover, Mineola, NY, 1998).

${ }^{23}$ D. L. Andrews, L. C. Dávila Romero, and G. E. Stedman, Phys. Rev. A 67, 055801 (2003)

${ }^{24}$ D. L. Andrews and L. C. Dávila Romero, Proc. SPIE 5218, 181 (2003).

${ }^{25}$ G. J. Daniels, R. D. Jenkins, D. S. Bradshaw, and D. L. Andrews, J. Chem. Phys. 119, 2264 (2003).

${ }^{26} \mathrm{R}$. Loudon, in The Quantum Theory of Light, 3rd ed. (University Press, Oxford, 2000), pp. 107-114.

${ }^{27}$ R. D. Jenkins and D. L. Andrews, Chem. Phys. Lett. 301, 235 (1999).

${ }^{28}$ D. L. Andrews, Spectrochim. Acta, Part A 46, 871 (1990).

${ }^{29}$ M. R. Shortreed, S. F. Swallen, Z.-Y. Shi, W. Tan, Z. Xu, C. Devadoss,
J. S. Moore, and R. Kopelman, J. Phys. Chem. B 101, 6318 (1997).

${ }^{30}$ S. F. Swallen, Z.-Y. Shi, W. Tan, Z. Xu, J. S. Moore, and R. Kopelman, J. Lumin. 76\&77, 193 (1998).

${ }^{31} \mathrm{Z} . \mathrm{Xu}$ and J. S. Moore, Acta Polym. 45, 83 (1994).

${ }^{32}$ M. Kimura, T. Shiba, T. Muto, K. Hanabusa, and H. Shirai, Macromolecules 32, 8237 (1999).

${ }^{33}$ M. S. Matos, J. Hofkens, W. Verheijen et al., Macromolecules 33, 2967 (2000).

${ }^{34}$ H. R. Stapert, N. Nishiyama, D.-L. Jiang, T. Aida, and K. Kataoka, Langmuir 16, 8182 (2000).

${ }^{35}$ H.-F. Chow, C.-F. Leung, G.-X. Wang, and Y.-Y. Yang, C. R. Chimie 6, 735 (2003).

${ }^{36}$ D. K. P. Ng, C. R. Chimie 6, 903 (2003).

${ }^{37}$ O. Finikova, A. Galkin, V. Rozhkov, M. Cordero, C. Hägerhäll, and S. Vinogradov, J. Am. Chem. Soc. 125, 4882 (2003).

${ }^{38}$ P. R. Hania, D. J. Heijs, T. Bowden, A. Pugžlys, J. van Esch, J. Knoester, and K. Duppen, J. Phys. Chem. B 108, 71 (2004).

${ }^{39}$ R. W. Wagner, T. E. Johnson, and J. S. Lindsey, J. Am. Chem. Soc. 118, 11166 (1996).

${ }^{40}$ M. del Rosario Benites, T. E. Johnson, S. Weghorn et al., J. Mater. Chem. 12, 65 (2002).

${ }^{41}$ J.-S. Hsiao, B. P. Krueger, R. W. Wagner et al., J. Am. Chem. Soc. 118, 11181 (1996).

${ }^{42}$ R. D. Jenkins and D. L. Andrews, J. Chem. Phys. 118, 3470 (2003).

${ }^{43}$ R. D. Jenkins and D. L. Andrews, Photochem. Photobiol. Sci. 2, 130 (2003).

${ }^{44}$ R. D. Jenkins and D. L. Andrews, Photochem. Photobiol. Sci. 3, 39 (2004).

${ }^{45}$ D. L. Andrews and G. Juzeliūnas, J. Chem. Phys. 96, 6606 (1992).

${ }^{46}$ D. L. Andrews and R. D. Jenkins, J. Chem. Phys. 114, 1089 (2001).

${ }^{47}$ R. D. Jenkins and D. L. Andrews, Phys. Chem. Chem. Phys. 2, 2837 (2000)

${ }^{48}$ M. Kawa and J. M. J. Fréchet, Chem. Mater. 10, 286 (1998).

${ }^{49}$ V. Vicinelli, P. Ceroni, M. Maestri, V. Balzani, M. Gorka, and F. Vögtle, J. Am. Chem. Soc. 124, 6461 (2002)

${ }^{50}$ M. Drobizhev, A. Rebane, C. Sigel, E. H. Elandaloussi, and C. W. Spangler, Chem. Phys. Lett. 325, 375 (2002).

${ }^{51}$ A. Dirksen and L. De Cola, C. R. Chimie 6, 873 (2003).

${ }^{52}$ C. Xu, W. Zipfel, J. B. Shear, R. M. Williams, and W. W. Webb, Proc. Natl. Acad. Sci. U.S.A. 93, 10763 (1996).

${ }^{53}$ K. König, J. Microsc. 200, 83 (2000).

${ }^{54}$ D. J. S. Birch, Spectrochim. Acta, Part A 57, 2313 (2001).

${ }^{55}$ M. G. Xu, B. Crimeen, M. J. Ludford-Menting, X. Gan, S. M. Russell, and M. Gu, Scanning 23, 9 (2001).

${ }^{56}$ G. F. White, K. L. Litvinenko, S. R. Meech, D. L. Andrews, and A. J. Thomson, Photochem. Photobiol. Sci. 3, 47 (2004).

${ }^{57}$ M. Halim, J. N. G. Pillow, I. D. W. Samuel, and P. L. Burn, Synth. Met. 102, 922 (1999).

${ }^{58}$ M. Halim, I. D. W. Samuel, J. N. G. Pillow, and P. L. Burn, Synth. Met. 102, 1113 (1999).

${ }^{59}$ A. W. Freeman, S. C. Koene, P. R. L. Malenfant, M. E. Thompson, and J. M. J. Fréchet, J. Am. Chem. Soc. 122, 12385 (2000).

${ }^{60}$ T. D. Anthopoulos, J. P. J. Markham, E. B. Namdas, J. R. Lawrence, I. D. W. Samuel, S.-C. Lo, and P. L. Burn, Org. Electron. 4, 71 (2003).

${ }^{61}$ P. Furuta, J. Brooks, M. E. Thompson, and J. M. J. Fréchet, J. Am. Chem. Soc. 125, 13165 (2003).

${ }^{62}$ N. Nishiyama, H. R. Stapert, G.-D. Zhang, D. Takasu, D.-L. Jiang, T. Nagano, T. Aida, and K. Kataoka, Bioconjugate Chem. 14, 58 (2003).

${ }^{63}$ G.-D. Zhang, N. Nishiyama, A. Harada, D.-L. Jiang, T. Aida, and K. Kataoka, Macromolecules 36, 1304 (2003).

${ }^{64}$ G.-D. Zhang, A. Harada, N. Nishiyama, D.-L. Jiang, H. Koyama, T. Aida, and K. Kataoka, J. Controlled Release 93, 141 (2003).

${ }^{65}$ V. Percec, W.-D. Cho, and G. Ungar, J. Am. Chem. Soc. 122, 10273 (2000).

${ }^{66}$ J. R. Parquette, C. R. Chimie 6, 779 (2003).

${ }^{67}$ J. M. J. Fréchet, J. Polym. Sci. A 41, 3713 (2003).

${ }^{68}$ S. Thayumanavan, P. Bharathi, K. Sivanandan, and D. R. Vutukuri, C. R. Chimie 6, 767 (2003). 